\title{
Intact survival from severe cardiogenic shock caused by the first attack of atrial tachycardia treated with extracorporeal membrane oxygenation and surgical left atrium appendage resection: a case report
}

\author{
Tatsuhiko Shimizu, Tomoyuki Kanazawa ${ }^{*}$, Takanobu Sakura, Kazuyoshi Shimizu and Tatsuo Iwasaki
}

\begin{abstract}
Background: Atrial tachycardia (AT) is rare in children and can usually be reversed to sinus rhythm with pharmacotherapy and cardioversion. We report a rare case of severe left-sided heart failure due to refractory AT.

Case presentation: A 12-year-old boy had AT with a heart rate of 180 beats/minute, which was refractory to any medication and defibrillation despite the first attack. Due to rapid cardiorespiratory collapse shortly after arriving at our hospital, central extracorporeal membrane oxygenation (ECMO) with left arterial venting was started immediately. Although AT persisted after that, it stopped on the 3rd day after admission following surgical resection of the left atrial appendage thought to be the source of AT. He was weaned off ECMO on the 7th day and ventilator on the 14th day.

Conclusions: The appropriate timing of central ECMO and surgical ablation were effective in saving this child from a life-threatening situation caused by refractory AT.
\end{abstract}

Keywords: Focal atrial tachycardia, Central extracorporeal membrane oxygenation, Surgical ablation

\section{Background}

Atrial tachycardia (AT) is one of the rare diseases in pediatric patients [1]. AT attack is treated by antiarrhythmic medication such as adenosine triphosphate (ATP), amiodarone, and $\beta$-blocker. Some patients have refractory AT that does not respond well to usual medical treatment. Long-lasting AT may cause cardiomyopathy when medical treatment would not effect for a long time. Extracorporeal membrane oxygenation (ECMO) support is occasionally necessary for hemodynamic support in severe cardiogenic shock with refractory AT [2].

\footnotetext{
${ }^{*}$ Correspondence: tyskanazawa@gmail.com

Department of Anesthesiology and Resuscitology, Okayama University Graduate School of Medicine, Dentistry and Pharmaceutical Sciences, 2-5-1 Shikata-cho, Kita-ku, Okayama 700-8558, Japan
}

Catheter ablation is one of the treatments for AT [3], especially when medication would not be effective. Furthermore, surgical resection of AT focus may be considered after failed catheter ablation $[4,5]$. We experienced a child who had a severe cardiogenic shock with the first episode of AT attack. We were able to rescue him with ECMO support and surgical ablation.

\section{Case presentation}

A 12-year-old boy (height $145 \mathrm{~cm}$, weight $33 \mathrm{~kg}$ ) was diagnosed with AT of more than 170 beats per minute (bpm) at the first visit to a clinic. He had felt bad feelings for several days without any common cold symptoms. He was transported to our hospital by car with his family. At the emergency department (ED), he was alert, but his heart rate (HR) was $180 \mathrm{bpm}$ and his systolic blood 
pressure (sBP) was $80 \mathrm{mmHg}$. The initial ECG showed a narrow complex tachycardia (Fig. 1), and transthoracic echocardiography showed only $20 \%$ of left ventricular ejection fraction, suggesting cardiomyopathy [1]. We suspected tachycardia-induced cardiomyopathy rather than acute myocarditis based on the blood tests of CRP $0.08 \mathrm{mg} / \mathrm{dL}, \mathrm{WBC} 6240 / \mu \mathrm{L}, \mathrm{CK} 363 \mathrm{U} / \mathrm{L}$, and CK-MB $14 \mathrm{U} / \mathrm{L}$, no ST changes on ECG, and no pericardial effusion on echocardiography. His respiratory status was stable and $\mathrm{SpO}_{2}$ was maintained $100 \%$ in room air. We started amiodarone-loading $5 \mathrm{mg} / \mathrm{kg}$ intravenously, and kept continuous infusion at $10 \mu \mathrm{g} / \mathrm{kg} / \mathrm{min}$, following three times ATP administration (10mg, 15mg, 20mg) and twice of electric cardioversion (20J, 30J), which did not have any improvement. During the medical treatment at ED, sBP was going down around $60 \mathrm{mmHg}$, and
$\mathrm{SpO}_{2}$ was $90-96 \%$ on oxygen mask $\left(100 \% \mathrm{O}_{2}, 4 \mathrm{~L} / \mathrm{min}\right)$. His hemodynamic and respiratory status had been worsening at ED, we decided to transport him to the intensive care unit (ICU) after endotracheal intubation. After ICU admission, we inserted an arterial line via the right brachial artery and a central venous line via the left jugular vein. We started milrinone infusion at $0.2 \mu \mathrm{g} / \mathrm{kg} / \mathrm{min}$ and noradrenaline at $0.02 \mu \mathrm{g} / \mathrm{kg} / \mathrm{min}$. Subsequently, his sBP improved from 50 to $80 \mathrm{mmHg}$; however, AT with HR of 180-200 bpm was not improved. Therefore, we decided to establish veno-arterial (VA) ECMO support for hemodynamic and respiratory instability due to left ventricular failure leading to pulmonary edema (Fig. 2). We chose central ECMO rather than peripheral ECMO to ensure complete unloading left ventricle by inserting the left atrial venting cannula. Central VA ECMO via

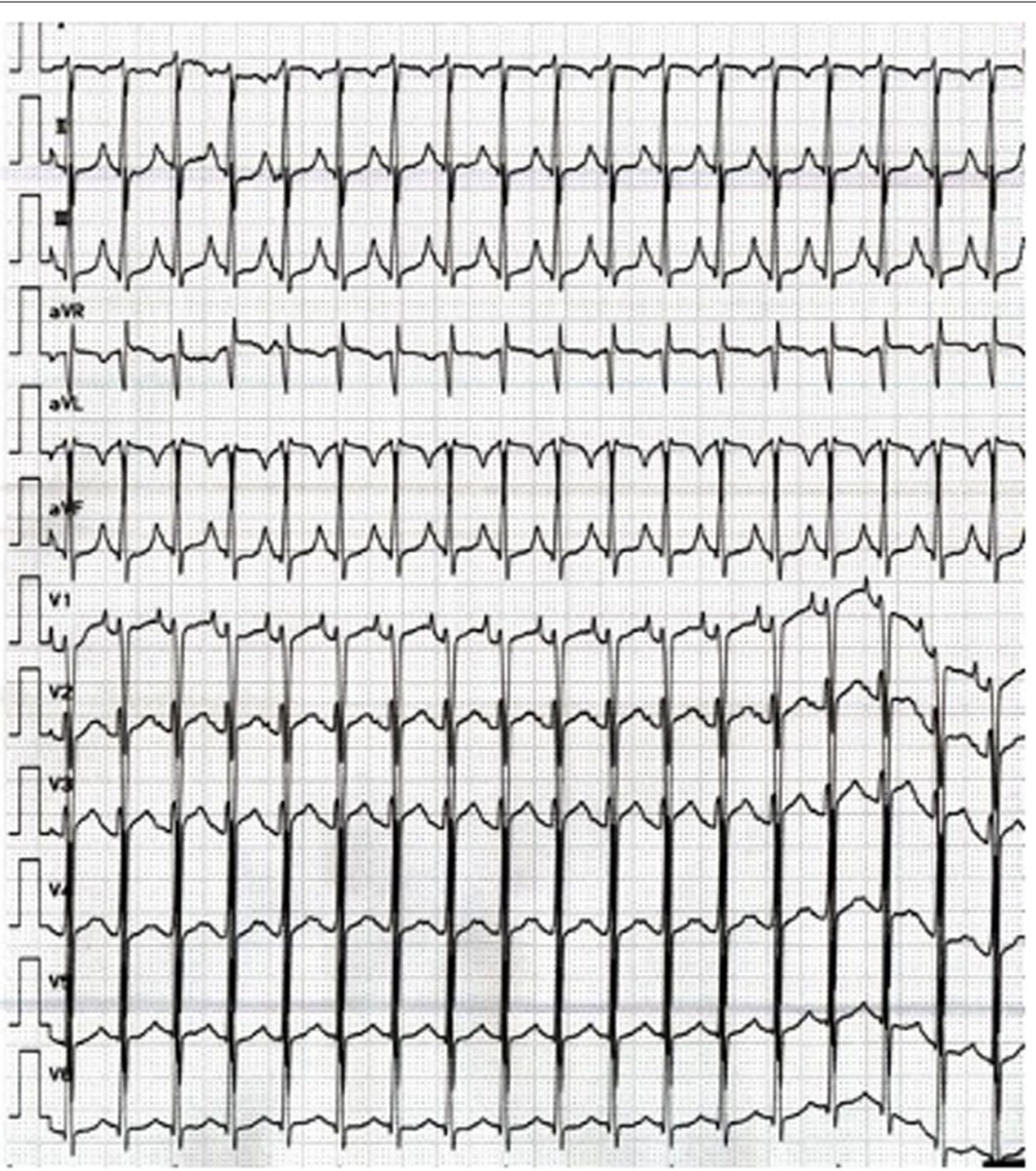

Fig. 1 Electrocardiogram on admission to ICU. Regular positive $P$ waves of 180-190 bpm and corresponding narrow QRS waves are seen in V1 and V2, although the $P$ wave is masked by the $T$ wave in most leads. 


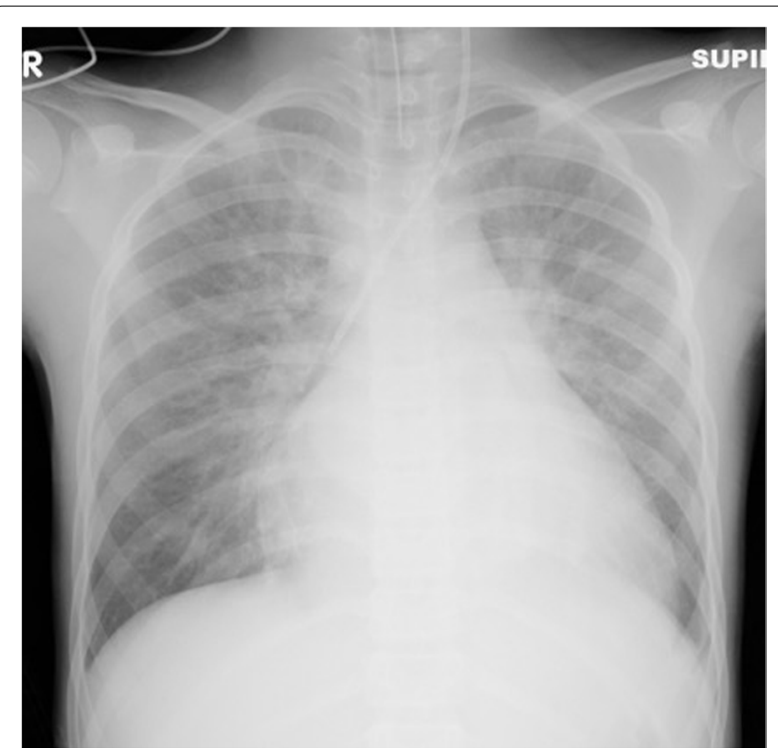

Fig. 2 Chest radiograph on admission to ICU

median sternotomy was uneventfully performed using 24-Fr right atrial, 20-Fr left atrial, and 16-Fr aortic cannula under general anesthesia with sevoflurane $1-1.5 \%$, fentanyl $500 \mu \mathrm{g}$, and rocuronium in the operating room (OR). Under $150 \mathrm{ml} / \mathrm{kg} / \mathrm{min}$ of ECMO flow support, we continued to provide amiodarone infusion up to $20 \mu \mathrm{g} /$ $\mathrm{kg} / \mathrm{min}$ and started continuous landiolol infusion up to $10 \mu \mathrm{g} / \mathrm{kg} / \mathrm{min}$. His HR was decreased to $140-160 \mathrm{bpm}$ several hours after ECMO induction and was settled down to 100-140 bpm on the 2nd clinical day; however, his cardiac rhythm was still AT. Although there was bleeding of 100-150 ml per hour under the control of ACT for around $150 \mathrm{~s}$ on the 2nd clinical day, the bleeding gradually settled down with blood transfusion as needed, and he underwent removal of intrathoracic hematoma in OR on the 4th clinical day. A pediatric cardiologist had diagnosed that the focus of his AT could be the left atrial appendage (LAA), based on ECG findings that the polarity of the $P$ wave was positive on V1, negative on I, positive on II, III, and aVF. Immediately after the cardiac surgeon clamped the base of the LAA according to the cardiologist's opinion, the patient's cardiac rhythm was reverted to sinus rhythm around 50-60 $\mathrm{bpm}$. The surgeon resected LAA as a surgical treatment of AT. After the surgical treatment, this child did not have an episode of AT during the ICU stay (Fig. 3) and we could completely discontinue amiodarone and landiolol infusion until the 5th clinical day. His cardiac function was dramatically improved, and we finally discontinued ECMO support on the 7th clinical day and milrinone on the 20th clinical day. He was extubated on the 14th clinical day. He was transported to the general ward without any antiarrhythmic medications on the 30th clinical day.

\section{Discussion}

We found two unique clinical viewpoints in this case. The first is the boy had been on central ECMO support for a sudden worsening of hemodynamic and respiratory status due to cardiomyopathy caused by refractory AT despite the first attack, and the second is he underwent surgical resection of LAA to treat AT on ECMO support.

Atrial tachycardia is rare in children. The median age at diagnosis is 7.2 years with the most commonly occurring from birth to 1 year, and most patients are treated with antiarrhythmic medications (ATP, amiodarone, $\beta$-blocker), cardioversion or catheter ablation [1]. Antiarrhythmic agents are well effective to recover normal sinus rhythm in most cases [1]; however, AT may cause cardiomyopathy when arrhythmic episode repeats frequently. When medical treatments as above would not be effective, mechanical hemodynamic support by ECMO should be considered for a rescue therapy because AT may cause cardiomyopathy when AT would not be recovered to sinus rhythm for a certain period of time $[2,6]$. Remarkably decreased left ventricular ejection fraction suggested cardiomyopathy, which required prompt treatment in this child. Several pediatric cases showed that ECMO support was performed for cardiomyopathy associated with refractory AT $[1,2]$ and refractory arrhythmias $[2,6-8]$. There have been no reports of patients requiring ECMO within a few days after the first attack. In this case, he had already had a severe cardiac failure on arrival at ED, which dramatically progressed leading to pulmonary edema despite treatment such as amiodarone infusion, bolus of ATP, and electric cardioversion. Our choice to induce ECMO as a rescue therapy for refractory AT was considered to be reasonable in this case. If we had hesitated to introduce ECMO, cardiac arrest and neurological sequelae would have been more likely to occur. In fact, Kang et al. reported a case of a 13-year-old girl who died of serious neurological complications after requiring ECMO due to circulatory collapse during the administration of amiodarone for treating refractory AT [1]. We also selected central ECMO with thoracotomy instead of peripheral ECMO. Although peripheral ECMO via inguinal approach does not require a thoracotomy, it not only increases left ventricular afterload, but also exacerbates pulmonary edema and delays recovery of left ventricular function due to inadequate left atrium (LA) decompression [9]. In contrast, central ECMO solves these problems, and Kotani et al. reported that ECMO with early LA decompression increases the rate of recovery of lung and left ventricular function [9]. After all, the 


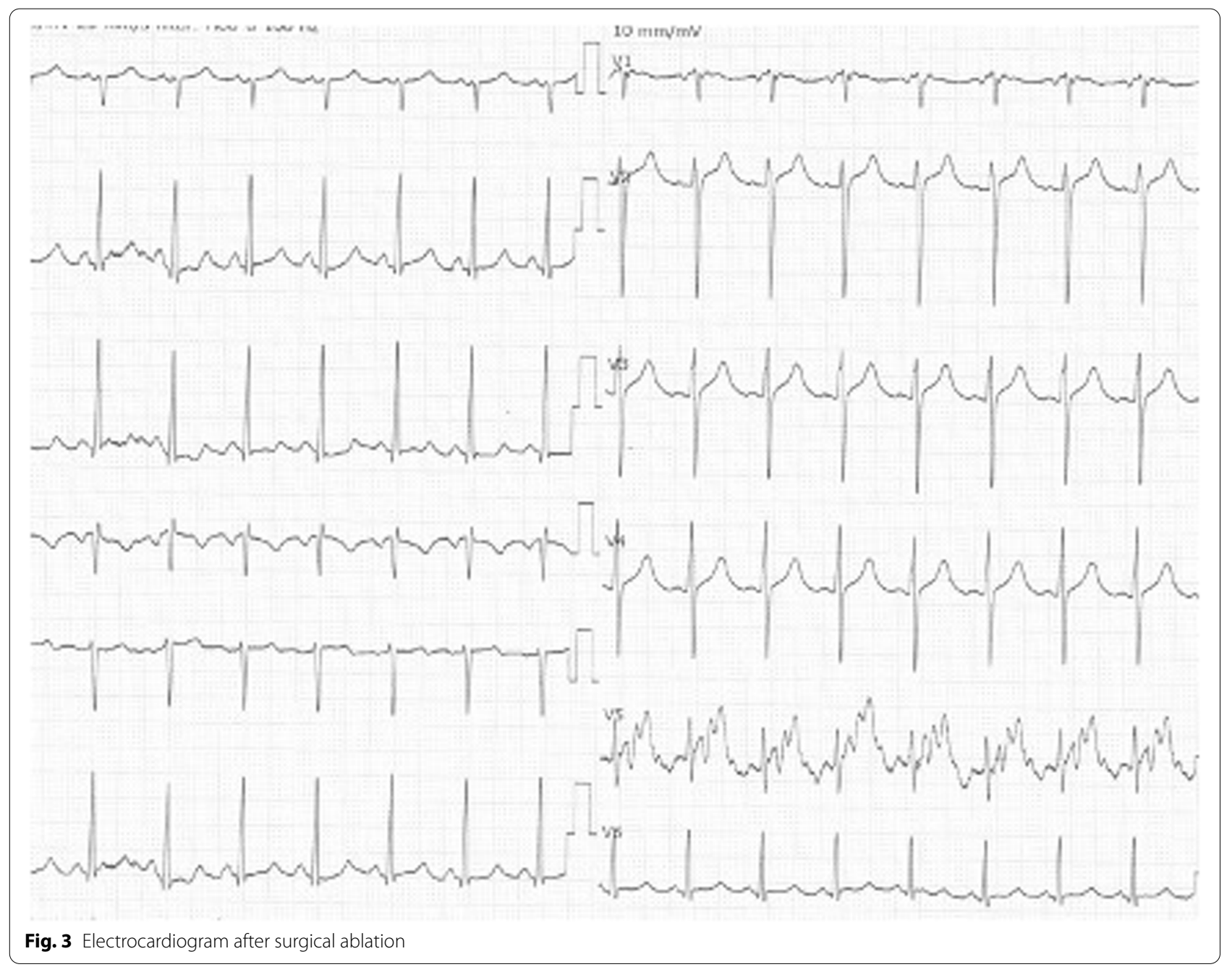

establishment of ECMO before the collapse contributed to the neurological prognosis, and the choice of central ECMO, which is possible to drainage blood from both atriums, instead of peripheral ECMO may have resulted in a faster weaning from ECMO and ventilator because of avoiding the damage to the heart and lungs [9]. Although bleeding complications are a greater problem with central ECMO than with peripheral ECMO, we think that the advantages of central ECMO outweighed the disadvantages of hemorrhage since the left ventricular function was significantly impaired in this case and peripheral ECMO could have worsened the condition of the heart and lungs due to increased left atrial and ventricular pressure with increased afterload.

In the second unique situation, surgical ablation was performed under ECMO support in this case. As a therapeutic option, catheter ablation is considered in the case of refractory AT, but it was difficult to perform catheter ablation in this case because of the rapid deterioration of his condition. Previous reports have shown that the atrial appendage is the source of focal AT in $11 \%$ of cases [1]. For refractory AT of LAA origin, Khan et al. reported a successful case of radiofrequency ablation on ECMO support [2], but Phillips et al. described that catheter ablation of focal AT resulting from LAA, especially distal point, was difficult due to the complex anatomy of LAA, and surgical appendectomy or thoracoscopic surgery may be required [10]. Pokushalov et al. reported a case of hemodynamically stable that ineffective catheter ablation followed by successful surgical ablation [4]. In this case, the chest had already been opened for central ECMO, and it was reasonable to perform surgical ablation during ECMO support as a rescue treatment because it was very difficult to plan catheter ablation in this situation. 
Finally, he was completely recovered from hemodynamic and respiratory failure by multidisciplinary treatment. ECMO support and surgical ablation played the main role in treating AT.

\section{Conclusion}

We had a rare case that the boy required ECMO support for severe hemodynamic and respiratory failure caused by refractory AT on the first attack. Adequate timing of ECMO induction and surgical treatment was effective to save children from a life-threatening situation caused by refractory AT.

\section{Abbreviations}

AT: Atrial tachycardia; ECMO: Extracorporeal membrane oxygenation; ATP: Adenosine triphosphate disodium hydrate; bpm: Beat per minute; ED: Emergency department; HR: Heart rate; sBP: Systolic blood pressure; ICU: Intensive care unit; VA: Veno-arterial; OR: Operating room; LAA: Left atrial appendage; LA: Left atrium.

\section{Acknowledgements}

Not applicable.

\section{Authors' contributions}

TS wrote the manuscript. TK obtained informed consent from parents, wrote the initial draft of the manuscript, and critically reviewed the manuscript. TS helped collect patient information. KS and TI provided critical revisions. The authors have approved the final version of the manuscript and have agreed to assume responsibility for all aspects of the work to ensure that any questions regarding the accuracy or completeness of any part of the work are properly investigated and resolved.

\section{Funding}

Not applicable.

\section{Availability of data and materials}

Not applicable.

\section{Declarations}

Ethics approval and consent to participate

In our institution, IRB approval is not required for a case report.

\section{Consent for publication}

We obtained written informed consent from the patient and his family for the publication of this case report in a scientific journal.

\section{Competing interests}

The authors declare that they have no competing interests.

Received: 15 July 2021 Revised: 7 October 2021 Accepted: 10 October 2021

Published online: 03 November 2021

\section{References}

1. Kang KT, Etheridge SP, Kantoch MJ, Tisma-Dupanovic S, Bradley DJ, Balaji $\mathrm{S}$, et al. Current management of focal atrial tachycardia in children a multicenter experience. Circ Arrhythm Electrophysiol. 2014;7:664-70.

2. Khan M, Gauri A, Grifka R, Elmouchi D. Radiofrequency ablation of a left atrial appendage tachycardia on ECMO support. Case Rep Pediatr. 2013. https://doi.org/10.1155/2013/203241.

3. Walsh EP, Saul JP, Hulse JE, et al. Transcatheter ablation of ectopic atrial tachycardia in young patients using radiofrequency current. Circulation. 1992;86:1138-46.

4. Pokushalov E, Romanov A, Artyomenko S, Rhodes LA, Hordof AJ, Mayer $J$ E, et al. Left atrial appendectomy after failed catheter ablation of a focal atrial tachycardia originating in the left atrial appendage. Pediatr Cardiol. 2010;31:908-11.

5. Torok RD, Wei B, Kanter RJ, Jaquiss RD, Lodge AJ. Thoracoscopic resection of the left atrial appendage after failed focal atrial tachycardia ablation. Ann Thorac Surg. 2014;97:1322-7.

6. Dyamenahalli U, Tuzcu V, Fontenot E, Papagiannis J, Jaquiss RD, Bhutta A, et al. Extracorporeal membrane oxygenation support for intractable primary arrhythmias and complete congenital heart block in newborns and infants: short-term and medium-term outcomes. Pediatr Crit Care Med. 2012;13:47-52.

7. Shebani SO, Ng GA, Stafford P, Duke C. Radiofrequency ablation on venoarterial extracorporeal life support in treatment of very sick infants with incessant tachymyopathy. Europace. 2015;17:622-7.

8. Boscamp NS, Turner ME, Crystal M, Anderson B, Vincent JA, Torres AJ. Cardiac catheterization in pediatric patients supported by extracorporeal membrane oxygenation: a 15-year experience. Pediatr Cardiol. 2017;38:332-7

9. Kotani Y, Chetan D, Rodrigues W, Sivarajan VB, Gruenwald C, Guerguerian $\mathrm{AM}$, et al. Left atrial decompression during venoarterial extracorporeal membrane oxygenation for left ventricular failure in children: current strategy and clinical outcomes. Artif Organs. 2013;37:29-36.

10. Phillips KP, Natale A, Sterba R, Saliba WI, Burkhardt JD, Wazni O, et al. Percutaneous pericardial instrumentation for catheter ablation of focal atrial tachycardias arising from the left atrial appendage. J Cardiovasc Electrophysiol. 2008;19:430-3.

\section{Publisher's Note}

Springer Nature remains neutral with regard to jurisdictional claims in published maps and institutional affiliations.

\section{Submit your manuscript to a SpringerOpen ${ }^{\odot}$ journal and benefit from:}

- Convenient online submission

- Rigorous peer review

- Open access: articles freely available online

- High visibility within the field

- Retaining the copyright to your article

Submit your next manuscript at springeropen.com 